\title{
General Classical Electrodynamics
}

\author{
Koen J. van Vlaenderen
}

\author{
Ethergy B.V, Research, Hobbemalaan 10, 1816GD Alkmaar, North Holland, The Netherlands \\ Institute for Basic Research, P.O. Box 1577, Palm Harbor, FL 34684, USA
}

Copyright (C) 2016 by authors, all rights reserved. Authors agree that this article remains permanently

open access under the terms of the Creative Commons Attribution License 4.0 International License

\begin{abstract}
Maxwell's Classical Electrodynamics (MCED) suffers several inconsistencies: (1) the Lorentz force law of MCED violates Newton's Third Law of Motion (N3LM) in case of stationary and divergent or convergent current distributions; (2) the general Jefimenko electric field solution of MCED shows two longitudinal far fields that are not waves; (3) the ratio of the electrodynamic energy-momentum of a charged sphere in uniform motion has an incorrect factor of $\frac{4}{3}$. A consistent General Classical Electrodynamics (GCED) is presented that is based on Whittaker's reciprocal force law that satisfies N3LM. The Whittaker force is expressed as a scalar magnetic field force, added to the Lorentz force. GCED is consistent only if it is assumed that the electric potential velocity in vacuum, ' $a$ ', is much greater than ' $c$ ' $(a \gg c)$; GCED reduces to MCED, in case we assume $a=c$. Longitudinal electromagnetic waves and superluminal longitudinal electric potential waves are predicted. This theory has been verified by seemingly unrelated experiments, such as the detection of superluminal Coulomb fields and longitudinal Ampère forces, and has a wide range of electrical engineering applications.
\end{abstract}

Keywords Classical Electrodynamics, Longitudinal Ampère Force, Scalar Fields, Longitudinal Electric Waves, Superluminal Velocity, Energy Conversion

\section{Introduction}

An alternative to Maxwell's [1, 2] Classical Electrodynamics (MCED) theory is presented, called General Classical Electrodynamics (GCED), that is free of inconsistencies. For the development of this theory we make use of the fundamental theorem of vector algebra. The proof of this fundamental theorem is based on the three dimensional delta function $\delta(\mathbf{x})$ and the sifting property of this function, see $(1.1-1.2)$ :

$$
\begin{aligned}
\delta(\mathbf{x}) & =\frac{-1}{4 \pi} \Delta\left(\frac{1}{|\mathbf{x}|}\right) \\
\mathbf{F}(\mathbf{x}) & =\int_{V^{\prime}} \mathbf{F}\left(\mathbf{x}^{\prime}\right) \delta\left(\mathbf{x}-\mathbf{x}^{\prime}\right) \mathrm{d}^{3} x^{\prime}
\end{aligned}
$$

The fundamental theorem of vector algebra is as follows: a vector function $\mathbf{F}(\mathbf{x})$ can be decomposed into two unique vector functions $\mathbf{F}_{l}(\mathbf{x})$ and $\mathbf{F}_{t}(\mathbf{x})$, such that

$$
\begin{aligned}
\mathbf{F}(\mathbf{x}) & =\mathbf{F}_{l}(\mathbf{x})+\mathbf{F}_{t}(\mathbf{x}) \\
\mathbf{F}_{l}(\mathbf{x}) & =-\frac{1}{4 \pi} \nabla \int_{V^{\prime}} \frac{\nabla^{\prime} \cdot \mathbf{F}\left(\mathbf{x}^{\prime}\right)}{\left|\mathbf{x}-\mathbf{x}^{\prime}\right|} \mathrm{d}^{3} x^{\prime} \\
\mathbf{F}_{t}(\mathbf{x}) & =\frac{1}{4 \pi} \nabla \times \int_{V^{\prime}} \frac{\nabla^{\prime} \times \mathbf{F}\left(\mathbf{x}^{\prime}\right)}{\left|\mathbf{x}-\mathbf{x}^{\prime}\right|} \mathrm{d}^{3} x^{\prime}
\end{aligned}
$$

The lowercase subindexes ' $\mathrm{l}$ ' and 't' will have the meaning of longitudinal and transverse in this paper. The longitudinal vector function $\mathbf{F}_{l}$ is curl free $\left(\nabla \times \mathbf{F}_{l}=\mathbf{0}\right)$, and the transverse vector function $\mathbf{F}_{t}$ is divergence free $\left(\nabla \cdot \mathbf{F}_{t}=0\right)$. We assume that $\mathbf{F}$ is well behaved ( $\mathbf{F}$ is zero if $|\mathbf{x}|$ is infinite). Let us further introduce the following notations and definitions.

$$
\begin{array}{ll}
\rho & \text { Net electric charge density, in } \mathrm{C} / \mathrm{m}^{3} \\
\mathbf{J}=\mathbf{J}_{l}+\mathbf{J}_{t} & \text { Net electric current density, in } \mathrm{A} / \mathrm{m}^{2}
\end{array}
$$

$\Phi \quad$ Net electric charge (scalar) potential, in V

$\mathbf{A}=\mathbf{A}_{l}+\mathbf{A}_{t} \quad$ Net electric current (vector) potential,

$$
\text { in } \mathrm{V} \cdot \mathrm{s} / \mathrm{m}
$$

$$
\begin{array}{lr}
\mathbf{E}_{\Phi}=-\nabla \Phi & \text { Electric field, in V/m } \\
\mathbf{E}_{L}=-\partial_{t} \mathbf{A}_{l} & \text { Field induced divergent electric field } \\
\mathbf{E}_{T}=-\partial_{t} \mathbf{A}_{t} & \text { Field induced rotational electric field } \\
B_{\Phi}=-\partial_{t} \Phi & \text { Field induced scalar field, in V/s } \\
B_{L}=-\nabla \cdot \mathbf{A}_{l} & \text { Scalar magnetic field, in T }=\mathrm{V} \cdot \mathrm{s} / \mathrm{m}^{2} \\
\mathbf{B}_{T}=\nabla \times \mathbf{A}_{t} & \text { Vector magnetic field, in } \mathrm{T}=\mathrm{V} \cdot \mathrm{s} / \mathrm{m}^{2}
\end{array}
$$

$\begin{array}{ll}\phi_{0} \ll \epsilon_{0}^{2} \mu_{0} & \text { Polarizability of vacuum, in F. } \mathrm{s}^{2} / \mathrm{m}^{3} \\ \mu_{0} & \text { Permeability of vacuum: } 4 \pi 10^{-7} \mathrm{H} / \mathrm{m} \\ \epsilon_{0} & \text { Permittivity of vacuum: } 8.854^{-12} \mathrm{~F} / \mathrm{m}\end{array}$




$$
\begin{array}{rr}
(\mathbf{x}, t)=(x, y, z, t) & \text { Place and time coordinates } \\
\partial_{t}=\frac{\partial}{\partial t} & \text { Partial time differential } \\
\nabla=\left(\frac{\partial}{\partial x}, \frac{\partial}{\partial y}, \frac{\partial}{\partial z}\right) & \text { Del operator } \\
\Delta=\nabla \cdot \nabla & \text { Laplace operator } \\
\Delta \Phi=\nabla \cdot \nabla \Phi, & \Delta \mathbf{A}=\nabla \cdot \mathbf{A}-\nabla \times \nabla \times \mathbf{A}
\end{array}
$$

The permittivity, permeability and polarizability of vacuum are constants. The charge- and current density distributions, the potentials and the fields, are functions of place and not always functions of time. Time independent functions are called stationary or static functions. Basically there are three types of charge-current density distributions:
A. Current free charge
$\mathbf{J}=\mathbf{0}$
B. Stationary currents
$\partial_{t} \mathbf{J}=\mathbf{0}$
1. closed circuit
2. open circuit
$\partial_{t} \mathbf{J}=\mathbf{0} \wedge \nabla \cdot \mathbf{J}=\mathbf{0}$
$\partial_{t} \mathbf{J}=\mathbf{0} \wedge \nabla \cdot \mathbf{J} \neq \mathbf{0}$
C. Time dependent currents $\partial_{t} \mathbf{J} \neq \mathbf{0}$

The charge conservation law (also called 'charge continuity') is true for all types of charge-current density distributions:

$$
\frac{\partial \rho}{\partial t}+\nabla \cdot \mathbf{J}=0
$$

The physics of current free charge density distributions is called Electrostatics (ES): $\partial_{t} \rho=-\nabla \cdot \mathbf{0}=0$. The physics of stationary current density distributions $\left(\partial_{t} \mathbf{J}=\mathbf{0}\right)$ is called General Magnetostatics (GMS). A special case of GMS are divergence free current distributions $(\nabla \cdot \mathbf{J}=0)$, and this is widely called Magnetostatics (MS) in the scientific educational literature. In case of Magnetostatics, the charge density distribution has to be static as well: $\partial_{t} \rho=-\nabla \cdot \mathbf{J}=0$, such that the electric field and the magnetic field are both static.

The Maxwell-Lorentz force law satisfies Newton's third law of motion (N3LM) in case of Electrostatics and Magnetostatics, however, this force law violates N3LM in case of General Magnetostatics. A violation of N3LM means that momentum is not conserved by GMS systems, for which there is no experimental evidence! This remarkable inconsistency in classical physics is rarely mentioned in the scientific educational literature.

This is not the only problematic aspects of MCED. Jefimenko's electric field expression that is derived from MCED theory, shows two longitudinal electric field terms that do not interact by induction with other fields, therefore these electric fields cannot be field waves and nevertheless these electric fields fall off in magnitude by distance, as far fields, which is inconsistent. A third inconsistency is the problematic $\frac{4}{3}$ factor in the ratio of the electric energy and the electromagnetic momentum of a charged sphere. In the next sections we describe these related inconsistencies of MCED theory in more detail, and how to resolve them.

\section{General Magnetostatics}

Let $\mathbf{J}(\mathbf{x})$ be a stationary current distribution. The vector potential $\mathbf{A}(\mathbf{x})$ at place vector $\mathbf{x}$ is given by:

$$
\begin{aligned}
\mathbf{A}(\mathbf{x}) & =\frac{\mu_{0}}{4 \pi} \int_{V^{\prime}} \frac{\mathbf{J}\left(\mathbf{x}^{\prime}\right)}{r} \mathrm{~d}^{3} x^{\prime} \\
\mathbf{r} & =\mathbf{x}-\mathbf{x}^{\prime} \\
r & =\left|\mathbf{x}-\mathbf{x}^{\prime}\right|
\end{aligned}
$$

Since $\partial_{t} \mathbf{A}=\mathbf{0}$ for stationary currents, the electric field equals $\mathbf{E}=\mathbf{E}_{\Phi}=-\nabla \Phi$, such that the Gauss law for General Magnetostatics is given by

$$
\nabla \cdot \mathbf{E}_{\Phi}(\mathbf{x}, t)=\frac{1}{\epsilon_{0}} \rho(\mathbf{x}, t)
$$

The magnetostatic vector field $\mathbf{B}_{T}(\mathbf{x})$ is defined by BiotSavart's law as follows:

$$
\begin{aligned}
\mathbf{B}_{T}(\mathbf{x}) & =\nabla \times \mathbf{A}_{t}(\mathbf{x})=\nabla \times \mathbf{A}(\mathbf{x}) \\
& =-\frac{\mu_{0}}{4 \pi} \int_{V^{\prime}} \mathbf{J}\left(\mathbf{x}^{\prime}\right) \times \nabla\left(\frac{1}{r}\right) \mathrm{d}^{3} x^{\prime} \\
& =\frac{\mu_{0}}{4 \pi} \int_{V^{\prime}} \frac{1}{r^{3}}\left[\mathbf{J}\left(\mathbf{x}^{\prime}\right) \times \mathbf{r}\right] \mathrm{d}^{3} x^{\prime}
\end{aligned}
$$

The magnetic field is indeed static, since the current density is stationary. From the continuity of charge (1.6), and (2.2), it follows that $\mathbf{J}_{l}=-\epsilon_{0} \partial_{t}\left(\mathbf{E}_{\Phi}\right)$, hence the Ampère law for General Magnetostatics is as follows:

$$
\nabla \times \mathbf{B}_{T}-\epsilon_{0} \mu_{0} \frac{\partial \mathbf{E}_{\Phi}}{\partial t}=\mu_{0} \mathbf{J}
$$

\subsection{The Lorentz force}

The magnetic force density, $\mathbf{f}_{T}(\mathbf{x})$, that acts transversely on current density $\mathbf{J}(\mathbf{x})$ at place $\mathbf{x}$, is given by:

$$
\begin{aligned}
\mathbf{f}_{T}(\mathbf{x}) & =\mathbf{J}(\mathbf{x}) \times \mathbf{B}_{T}(\mathbf{x}) \\
& =\frac{\mu_{0}}{4 \pi} \int_{V^{\prime}} \frac{1}{r^{3}} \mathbf{J}(\mathbf{x}) \times\left[\mathbf{J}\left(\mathbf{x}^{\prime}\right) \times \mathbf{r}\right] \mathrm{d}^{3} x^{\prime} \\
& =\frac{\mu_{0}}{4 \pi} \int_{V^{\prime}} \frac{1}{r^{3}}\left[[\mathbf{J}(\mathbf{x}) \cdot \mathbf{r}] \mathbf{J}\left(\mathbf{x}^{\prime}\right)-\left[\mathbf{J}\left(\mathbf{x}^{\prime}\right) \cdot \mathbf{J}(\mathbf{x})\right] \mathbf{r}\right] \mathrm{d}^{3} x^{\prime} \\
& =\frac{\mu_{0}}{4 \pi} \int_{V^{\prime}} \mathbf{f}_{T}\left(\mathbf{x}, \mathbf{x}^{\prime}\right) \mathrm{d}^{3} x^{\prime}
\end{aligned}
$$

This is the Lorentz force density law for Magnetostatics; it is assumed that the electric force densities are negligible. Notice that the integrand is non-reciprocal: $\mathbf{f}_{T}\left(\mathbf{x}, \mathbf{x}^{\prime}\right) \neq$ $-\mathbf{f}_{T}\left(\mathbf{x}^{\prime}, \mathbf{x}\right)$, and that $\mathbf{r}$ changes into $-\mathbf{r}$ by swapping $\mathbf{x}$ and $\mathbf{x}^{\prime}$. This means that the Lorentz force law agrees with N3LM, but only if one calculates the total force on closed on-itself current circuits (magnetostatics is usually defined for divergence free currents only), which is proven as follows.

Consider two non-intersecting and closed current circuits $C$ and $C^{\prime}$, that carry the stationary electric currents $I$ and $I^{\prime}$, see figure 1 . The currents $I$ and $I^{\prime}$ are equal to the surface integral of the current density over a circuit line cross section of respectively circuits $C$ and $C^{\prime}$. The total force acting on circuit $C$ is a double volume integral of the Lorentz force density. We assume that the currents in $C$ and $C^{\prime}$ are constant for each circuit line cross section, therefore we replace the double volume integral by a double line integral over the circuits $C$ and $C^{\prime}$, in order to determine the force, $\mathbf{F}_{C}$, acting on $C$ : 


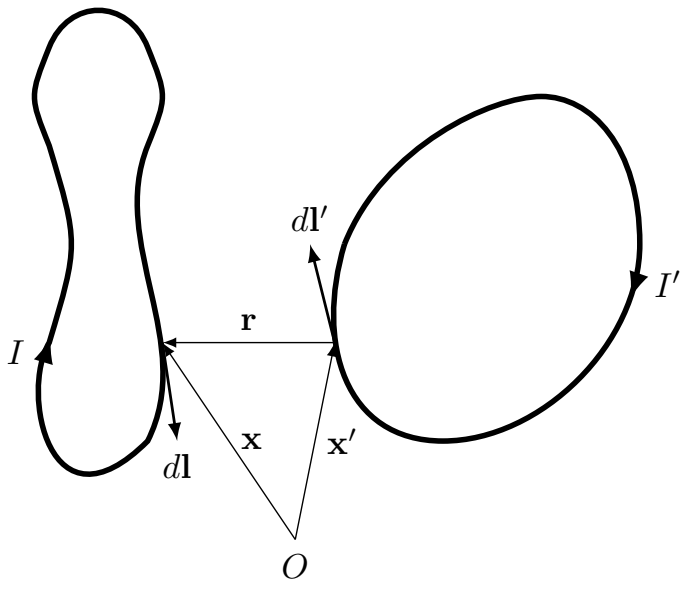

Figure 1. Closed stationary current circuits

$$
\begin{aligned}
\mathbf{F}_{C} & =-\frac{\mu_{0} I I^{\prime}}{4 \pi} \oint_{C} \oint_{C^{\prime}} d \mathbf{l} \times\left(d \mathbf{l}^{\prime} \times \nabla\left[\frac{1}{r}\right]\right) \\
& =-\frac{\mu_{0} I I^{\prime}}{4 \pi} \oint_{C^{\prime}} \oint_{C}\left[\left(d \mathbf{l} \cdot \nabla\left[\frac{1}{r}\right]\right) d \mathbf{l}^{\prime}-\left(d \mathbf{l} \cdot d \mathbf{l}^{\prime}\right) \nabla\left[\frac{1}{r}\right]\right] \\
& =\frac{\mu_{0} I I^{\prime}}{4 \pi} \oint_{C} \oint_{C^{\prime}}\left(d \mathbf{l} \cdot d \mathbf{l}^{\prime}\right) \nabla\left[\frac{1}{r}\right]
\end{aligned}
$$

This is Grassmann's [3] force law for closed current circuits. Since the curl of a gradient is zero, the first integral disappears, see (2.6) after the first derivation. The final integral after derivation step 2 has a reciprocal integrand, such that the force acting on circuit $C^{\prime}$ is the exact opposite of the force acting on circuit $C\left(\mathbf{F}_{C}=-\mathbf{F}_{C^{\prime}}\right)$, in agreement with N3LM. Fubini's theorem is applicable in derivation step 1 (switching the integration order in the first integral), since it is assumed the circuits $C$ and $C^{\prime}$ do not intersect.

The standard literature on Classical Electrodynamics usually defines Magnetostatics as the physics of stationary and divergence free (closed circuits) electric currents. For example, the Feynman lectures [4, Vol II, $\S 13.4]$ describe that Magnetostatics is based on equation $\nabla \times \mathbf{B}_{T}=\mu \mathbf{J}$, such that Magnetostatic current is divergence free, such that the electric field is also static, and such that charge density is constant in time: $\nabla \cdot \mathbf{J}=0=\partial_{t} \rho$. R. Feynman further suggests that a Magnetostatic circuit may contain batteries or generators that keep the charges flowing, however, an electric battery delivers an electrical current only if the battery's charge density changes in time. A generator of stationary current is for example Faraday's homopolar disk generator, which can be included in a stationary closed current loop. One has to measure the force $\mathbf{F}_{C}$ on the entire closed circuit that includes the generator as well, while the generator is externally driven with constant speed. Such a magnetostatic force experiment has yet to be done. J. D. Jackson's [5] third edition of Classical Electrodynamics postulates without proof that $\nabla \cdot \mathbf{J}=0=\partial_{t} \rho$ (charge density is time-independent anywhere in space, see after equation 5.3) before Jackson treats the laws of magnetostatics. D. J. Griffiths' [6] treatment of magnetostatics is likewise: "stationary electric currents are such that the density of charge is constant anywhere", which means that stationary currents that are divergence free. A. Altland [7] defined 'statics' as 'static electric fields and static magnetic fields', and again this implies that $\nabla \cdot \mathbf{J}=0=\partial_{t} \rho$.
Etc ...

It is not at all straightforward to find practical examples of a measured force exerted on a stationary and perfectly closedon-itself current circuit. The Meisner effect might be such an example: a free falling permanent magnet approaches a superconductor, which induces a closed-circuit current in the superconductor. The magnet falls until the induced currents and magnetic field of the superconductor perfectly opposes the field of the magnet, which causes the magnet to levitate, and from that moment on the superconductor current is stationary and divergence free. However, we cannot measure the "electric current" of the floating permanent magnet, in order to derive a magnetostatics force law. An electrically charged rotating object may represent a closed-circuit stationary current, however, it seems impractical to measure forces on such objects while keeping the rotation speed constant during the measurements. Ampère force experiments with two coils that conduct stationary currents have been performed frequently in history. A coil with several windings gives the impression of a perfectly closed current loop, however, this is only true approximately.

Many stationary current experiments have been conducted to measure the Ampère force on a circuit that is not closedon-itself, see figure 2 .

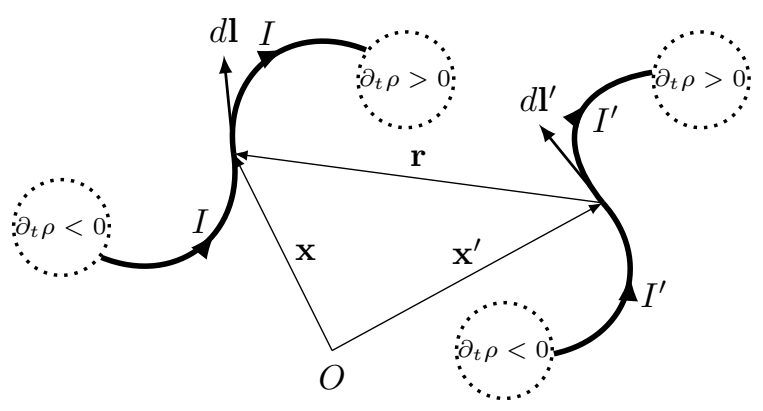

Figure 2. Open stationary current circuits

One can perform such experiments by applying two sliding contacts in order to enable a rotation- or translation motion of a circuit part that is non-closed, such that a force can be measured on just this movable circuit part. Faraday's homopolar disk motor is an example of this principle. Stefan Marinov's [8] Siberian Coliu motor is another example of a two sliding contacts motor, driven by a stationary current. Another type of non-closed current circuits make use of light weight movable batteries, such that sliding contacts can be avoided. This shows that the condition $\nabla \cdot \mathbf{J}=0=\partial_{t} \rho$, as well as the conditions $\nabla \times \mathbf{B}=\mu \mathbf{J}$ and $\partial_{t} \mathbf{E}=\mathbf{0}$, are artificial and superfluous for force experiments on stationary electric currents.

A possible reason for reducing General Magnetostatics to Magnetostatics by means of the false assumption that stationary currents are divergence free in general, is to obscure mathematically the violation of N3LM by the Grassmann force law [9], and also by the more general Lorentz force law. General Magnetostatics may be consistent with Classical Mechanics, if the Lorentz force law is replaced by another force law that satisfies N3LM.

\subsection{The Whittaker force}

J.C. Maxwell [2, Part IV, Chapter II, p.174] actually preferred Ampère's original force law to Grassmann's force law, because of Newton's third law of motion. An analysis of 
Ampère's force law by E. T. Whittaker [10, p.91], resulted in the following Whittaker force law,

$$
\left.\mathbf{F}_{C}=\frac{\mu_{0} I I^{\prime}}{4 \pi} \int_{C} \int_{C^{\prime}} \frac{1}{r^{3}}\left[\left(d \mathbf{l}^{\prime} \cdot \mathbf{r}\right) d \mathbf{l}+(d \mathbf{l} \cdot \mathbf{r}) d \mathbf{l}^{\prime}-\left(d \mathbf{l} \cdot d \mathbf{l}^{\prime}\right) \mathbf{r}\right)\right]
$$

that is equal to Grassmann's force law (2.6), except for the additional term $\left(d \mathbf{l}^{\prime} \cdot \mathbf{r}\right) d \mathbf{l}$. Both force laws predict the same force acting on closed on-itself circuits, since the line integral of the additional term over a closed circuit disappears as well. However, Whittaker's force law is reciprocal $\left(\mathbf{F}_{C}=-\mathbf{F}_{C^{\prime}}\right)$, also for non-closed circuits, and satisfies N3LM for General Magnetostatics.

By means of the following functions, defined as follows,

$$
\begin{aligned}
B_{L}(\mathbf{x}) & =-\nabla \cdot \mathbf{A}_{l}(\mathbf{x})=-\nabla \cdot \mathbf{A}(\mathbf{x}) \\
& =-\frac{\mu_{0}}{4 \pi} \int_{V^{\prime}} \mathbf{J}\left(\mathbf{x}^{\prime}\right) \cdot \nabla\left(\frac{1}{r}\right) \mathrm{d}^{3} x^{\prime} \\
& =\frac{\mu_{0}}{4 \pi} \int_{V^{\prime}} \frac{1}{r^{3}}\left[\mathbf{J}\left(\mathbf{x}^{\prime}\right) \cdot \mathbf{r}\right] \mathrm{d}^{3} x^{\prime} \\
\mathbf{f}_{L}(\mathbf{x}) & =\mathbf{J}(\mathbf{x}) B_{L}(\mathbf{x})
\end{aligned}
$$

we generalize Whittaker's force law as a double volume integral of field force densities, see [11, equation 13], and [12]:

$$
\begin{gathered}
\int_{V}\left[\mathbf{f}_{L}(\mathbf{x})+\mathbf{f}_{T}(\mathbf{x})\right] \mathrm{d}^{3} x= \\
\int_{V}\left[\mathbf{J}(\mathbf{x}) B_{L}(\mathbf{x})+\mathbf{J}(\mathbf{x}) \times \mathbf{B}_{T}(\mathbf{x})\right] \mathrm{d}^{3} x= \\
\frac{\mu_{0}}{4 \pi} \int_{V} \int_{V^{\prime}} \frac{1}{r^{3}}\left[\left[\mathbf{J}\left(\mathbf{x}^{\prime}\right) \cdot \mathbf{r}\right] \mathbf{J}(\mathbf{x})+[\mathbf{J}(\mathbf{x}) \cdot \mathbf{r}] \mathbf{J}\left(\mathbf{x}^{\prime}\right)-\right. \\
\left.\left[\mathbf{J}\left(\mathbf{x}^{\prime}\right) \cdot \mathbf{J}(\mathbf{x})\right] \mathbf{r}\right] \mathrm{d}^{3} x^{\prime} \mathrm{d}^{3} x
\end{gathered}
$$

This double volume integral of force densities satisfies N3LM for stationary current densities in general, since the integrand is reciprocal. The additional force density, $\mathbf{f}_{L}$, is called the longitudinal Ampère force density, which balances the transverse Ampère force density, $\mathbf{f}_{T}$, such that the total Ampère force density $\mathbf{f}(\mathbf{x})=\mathbf{f}_{L}(\mathbf{x})+\mathbf{f}_{T}(\mathbf{x})$ satisfies $\mathbf{f}(\mathbf{x})=-\mathbf{f}\left(\mathbf{x}^{\prime}\right)$, see figure 3 .

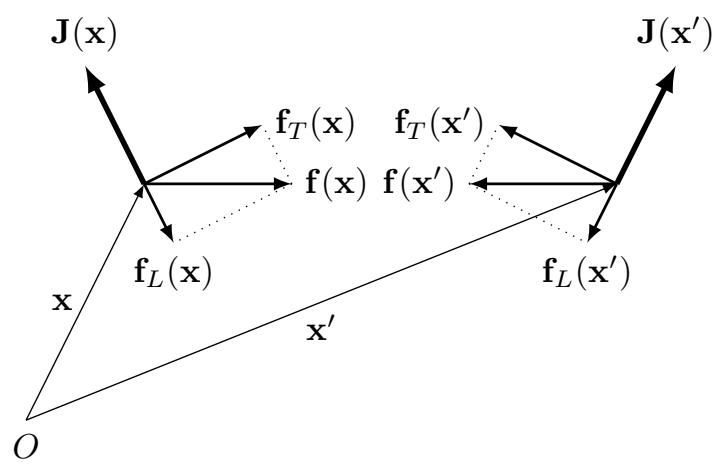

Figure 3. Total Ampère force density

It is obvious that the scalar function $B_{L}$ is a physical field that mediates an observable Ampère force, just like the vector magnetic field $\mathbf{B}_{T}$, and therefore it is called the scalar magnetic field [13].

\subsection{The Lorenz condition}

By means of (1.1), (1.2) and (2.3), (2.8), we derive the following equations:

$$
\begin{aligned}
\nabla B_{L}(\mathbf{x})+\nabla \times \mathbf{B}_{T}(\mathbf{x}) & =-\Delta \mathbf{A}(\mathbf{x}) \quad=\mu_{0} \mathbf{J}(\mathbf{x}) \\
\nabla B_{L}(\mathbf{x}) & =-\nabla \nabla \cdot \mathbf{A}_{l}(\mathbf{x})=\mu_{0} \mathbf{J}_{l}(\mathbf{x}) \\
\nabla \times \mathbf{B}_{T}(\mathbf{x}) & =\nabla \times \nabla \times \mathbf{A}_{t}(\mathbf{x})=\mu_{0} \mathbf{J}_{t}(\mathbf{x})
\end{aligned}
$$

The following GMS condition follows from (2.4), (2.11) and (3.1), and is known as the Lorenz condition [14]:

$$
\nabla\left(B_{L}+\epsilon_{0} \mu_{0} B_{\Phi}\right)=0
$$

This GMS condition is not a free to choose "gauge" condition. If the scalar function $B_{L}$ has the meaning of a physical field, then Lorenz's condition shows that the scalar function $B_{\Phi}$ also has the meaning of a physical field. So far we have shown a consistent GMS theory in agreement with classical mechanics.

\section{General CED}

We continue to develop the theory of General CED that describes the physics of time dependent currents, taking into account the physical scalar fields $B_{L}, B_{\Phi}$ and (2.2), (2.12) and (2.13).

\subsection{General field induction}

The field equations that describe the induction of secondary fields by primary time dependent fields, follow directly from the field definitions and the fact that the operators $\nabla, \nabla \cdot$ and $\nabla \times$ commute with $\partial_{t}$ :

$$
\begin{aligned}
\nabla B_{\Phi}-\frac{\partial \mathbf{E}_{\Phi}}{\partial t} & =-\nabla \frac{\partial \Phi}{\partial t}+\frac{\partial(\nabla \Phi)}{\partial t}=\mathbf{0} \\
\nabla \cdot \mathbf{E}_{L}-\frac{\partial B_{L}}{\partial t} & =-\nabla \cdot \frac{\partial \mathbf{A}_{l}}{\partial t}+\frac{\partial\left(\nabla \cdot \mathbf{A}_{l}\right)}{\partial t}=0 \\
\nabla \times \mathbf{E}_{T}+\frac{\partial \mathbf{B}_{T}}{\partial t} & =-\nabla \times \frac{\partial \mathbf{A}_{t}}{\partial t}+\frac{\partial\left(\nabla \times \mathbf{A}_{t}\right)}{\partial t}=\mathbf{0}
\end{aligned}
$$

This is the generalization of Faraday's [15] law of induction, see (3.3). A curl-free electric field $\mathbf{E}_{L}$ is induced by a time varying scalar magnetic field $B_{L}$, see (3.2), which is similar to the Faraday induction of a divergence-free electric field $\mathbf{E}_{T}$. Equation (3.2) will be called Nikolaev's [13] law of electromagnetic induction, after G.V. Nikolaev.

Electric fields are sourced by static charges and induced by time varying vector- and scalar magnetic fields. According to the superposition principle, a superimposed electric field is defined as $\mathbf{E}=\mathbf{E}_{\Phi}+\mathbf{E}_{L}+\mathbf{E}_{T}=-\nabla \Phi-\partial_{t} \mathbf{A}$. Notice that $\mathbf{E}_{l}=\mathbf{E}_{\Phi}+\mathbf{E}_{L}$ and that $\mathbf{E}_{t}=\mathbf{E}_{T}$.

\subsection{The essence of Maxwell's CED}

Maxwell's famous treatise on electricity and magnetism does not include the definitions of the fields $B_{L}$ and $B_{\Phi}$, and does not include Nikolaev's induction law. However, Maxwell defined the superimposed electric field as $\mathbf{E}=$ $\mathbf{E}_{\Phi}+\mathbf{E}_{L}+\mathbf{E}_{T}$, without explaining the induction of the secondary field $\mathbf{E}_{L}$ by a time varying primary field $B_{L}$. We cannot just assume the existence of field $\mathbf{E}_{L}$ without experimental proof, therefore the superimposed electric field may 
be defined as $\mathbf{E}=\mathbf{E}_{\Phi}+\mathbf{E}_{T}$, according to Ockham's razor. This simple definition reduces MCED to its experimental essence, and fixes the indeterminacy (gauge freedom) of the charge- and current potentials: the electric field $\mathbf{E}_{\Phi}+\mathbf{E}_{T}$ is not invariant with respect to the "gauge" potential transformation. The following field equations describe the essence of Maxwell's CED:

$$
\begin{aligned}
\mathbf{E}_{\Phi}+\mathbf{E}_{T} & =\mathbf{E} \\
\nabla \cdot \mathbf{E}=\nabla \cdot \mathbf{E}_{\Phi} & =\frac{1}{\epsilon_{0}} \rho \\
\nabla \times \mathbf{E}=\nabla \times \mathbf{E}_{T} & =-\frac{\partial \mathbf{B}_{T}}{\partial t} \\
\nabla \times \mathbf{B}_{T}-\epsilon_{0} \mu_{0} \frac{\partial\left(\mathbf{E}_{\Phi}+\mathbf{E}_{T}\right)}{\partial t} & =\mu_{0} \mathbf{J}
\end{aligned}
$$

Completed with the Lorentz force law, this theory is called Special Classical Electrodynamics (SCED). From the charge continuity law follows the next 'displacement current' equation:

$$
-\epsilon_{0} \frac{\partial \mathbf{E}_{\Phi}}{\partial t}=\mathbf{J}_{l}
$$

Substraction of (3.8) from (3.7) gives the following equation.

$$
\nabla \times \mathbf{B}_{T}-\epsilon_{0} \mu_{0} \frac{\partial \mathbf{E}_{T}}{\partial t}=\mu_{0} \mathbf{J}_{t}
$$

The presence of Maxwell's displacement current term $\epsilon_{0} \partial_{t} \mathbf{E}_{T}$ in the Maxwell-Ampère law (3.7) and in (3.9) does not follow directly from charge continuity, since this term is divergence free. The addition of this term by Maxwell was an unfounded conjecture, which led to the derivation of the transverse electromagnetic (TEM) wave equations. Maxwell's conjecture was eventually proven and verified by Hertz's [16] electromagnetic wave detection experiments.

Rewriting (3.5) and (3.9) in terms of the potentials gives:

$$
\begin{aligned}
-\Delta \Phi & =\frac{1}{\epsilon_{0}} \rho \\
\epsilon_{0} \mu_{0} \frac{\partial^{2} \mathbf{A}_{t}}{\partial t^{2}}+\nabla \times \nabla \times \mathbf{A}_{t} & =\mu_{0} \mathbf{J}_{t}
\end{aligned}
$$

The derivation of these 'decoupled' differential equations for the potentials is independent of gauge conditions and gauge transformations. The indeterminacy (gauge freedom) of the potentials is a direct consequence of Maxwell's unfounded addition of the field $\mathbf{E}_{L}$ to the superimposed electric field, within the context of MCED. These equations have the following solutions.

$$
\begin{aligned}
\Phi(\mathbf{x}, t) & =\frac{1}{4 \pi \epsilon_{0}} \int_{V^{\prime}} \frac{\rho\left(\mathbf{x}^{\prime}, t\right)}{r} \mathrm{~d}^{3} x^{\prime} \\
\mathbf{A}_{t}(\mathbf{x}, t) & =\frac{\mu_{0}}{4 \pi} \int_{V^{\prime}} \frac{\mathbf{J}_{t}\left(\mathbf{x}^{\prime}, t_{c}\right)}{r} \mathrm{~d}^{3} x^{\prime} \\
r & =\left|\mathbf{x}-\mathbf{x}^{\prime}\right| \quad c=\frac{1}{\sqrt{\epsilon_{0} \mu_{0}}} \\
t_{c} & =t-\frac{r}{c}
\end{aligned}
$$

The net charge potential $\Phi$ is instantaneous at a distance. The net current potential $\mathbf{A}_{t}$ is retarded with time interval $r / c$, relative to current potential sources at a distance $r$. We derive general field solutions, similar to the Jefimenko's fields, from the potential functions 3.12 and 3.13, and definition 3.4:

$$
\begin{aligned}
\mathbf{B}_{T}(\mathbf{x}, t) & =\frac{\mu_{0}}{4 \pi} \int_{V^{\prime}}\left[\frac{\mathbf{J}_{t}\left(\mathbf{x}^{\prime}, t_{c}\right) \times \mathbf{r}}{r^{3}}+\frac{\dot{\mathbf{j}}_{t}\left(\mathbf{x}^{\prime}, t_{c}\right) \times \mathbf{r}}{c r^{2}}\right] \mathrm{d}^{3} x^{\prime} \\
\mathbf{E}(\mathbf{x}, t) & =\frac{1}{4 \pi \epsilon_{0}} \int_{V^{\prime}}\left[\frac{\rho\left(\mathbf{x}^{\prime}, t\right) \mathbf{r}}{r^{3}}-\frac{\dot{\mathbf{J}}_{t}\left(\mathbf{x}^{\prime}, t_{c}\right)}{c^{2} r}\right] \mathrm{d}^{3} x^{\prime}
\end{aligned}
$$

Jefimenko's general fields are derived from MCED, and are the following expressions:

$$
\begin{aligned}
\mathbf{B}_{T}(\mathbf{x}, t)=\frac{\mu_{0}}{4 \pi} \int_{V^{\prime}}[ & \left.\frac{\mathbf{J}_{t}\left(\mathbf{x}^{\prime}, t_{c}\right) \times \mathbf{r}}{r^{3}}+\frac{\dot{\mathbf{J}}_{t}\left(\mathbf{x}^{\prime}, t_{c}\right) \times \mathbf{r}}{c r^{2}}\right] \mathrm{d}^{3} x^{\prime} \\
\mathbf{E}(\mathbf{x}, t)=\frac{1}{4 \pi \epsilon_{0}} \int_{V^{\prime}} & {\left[\frac{\rho\left(\mathbf{x}^{\prime}, t_{c}\right) \mathbf{r}}{r^{3}}+\frac{\dot{\rho}\left(\mathbf{x}^{\prime}, t_{c}\right) \mathbf{r}}{c r^{2}}\right.} \\
& \left.-\frac{\dot{\mathbf{J}}_{l}\left(\mathbf{x}^{\prime}, t_{c}\right)}{c^{2} r}-\frac{\dot{\mathbf{J}}_{t}\left(\mathbf{x}^{\prime}, t_{c}\right)}{c^{2} r}\right] \mathrm{d}^{3} x^{\prime}
\end{aligned}
$$

Jackson [17] proved that Jefimenko's field expressions do not depend on the choice for gauge condition. Notice that Jefimenko's magnetic field is expressed in terms of the divergence free current density, $\mathbf{J}_{t}$. The second term and third term of the integrand of (3.18) are longitudinal electric far fields that fall off in magnitude by $r$. Since far fields are field waves that can only exist as two fields that induce each other in turn, these two far field terms represent an inconsistency, since MCED does not define the two other fields that mutually induce the two longitudinal electric far fields that occur in Jefimenko's electric field expression. The two missing fields prove to be $B_{\Phi}$ and $B_{L}$, see $\S 3.3$. We call this the far field inconsistency of MCED.

SCED theory is manifestly far field consistent, since (3.15) and (3.16) only show the two far fields of the transverse electromagnetic wave. SCED is a consistent theory as special case of GCED with the condition $\mathbf{J}_{l}=\mathbf{0}$. If we assume $\mathbf{J}_{l}=\mathbf{0}$, then the second and third term in the integrand of (3.18) disappear, and in that case MCED and SCED are equal, except that the first integrand term of (3.18) is retarded, where the first integrand term of (3.16) is instantaneous. SCED and MCED describe the same physical effects in essence, if we ignore for a moment those experiments that determine the velocity of the near electric field.

\subsection{General displacement terms and general field waves}

We combine GMS with SCED in order to derive a consistent GCED. This theory should treat $\mathbf{J}_{l}$ and $\rho$ as the sources of fields $B_{L}, B_{\Phi}$ and $\mathbf{E}_{L}$, and should define the superimposed electric field as $\mathbf{E}=\mathbf{E}_{\Phi}+\mathbf{E}_{L}+\mathbf{E}_{T}$. We already generalized Faraday's field induction law in $\S 3.1$, and now we generalize Maxwell's conjectural term $\epsilon_{0} \partial_{t} \mathbf{E}_{T}$, by adding a displacement charge term $\phi_{0} \partial_{t} B_{\Phi}$ to(3.5) and a second displacement current term $\lambda_{0} \partial_{t} \mathbf{E}_{L}$ to (2.12):

$$
\begin{aligned}
& \nabla \cdot \mathbf{E}_{\Phi}-\frac{\phi_{0}}{\epsilon_{0}} \frac{\partial B_{\Phi}}{\partial t}=\frac{\phi_{0}}{\epsilon_{0}} \frac{\partial^{2} \Phi}{\partial t^{2}}-\nabla \cdot \nabla \Phi=\frac{1}{\epsilon_{0}} \rho \\
& \nabla B_{L}-\lambda_{0} \mu_{0} \frac{\partial \mathbf{E}_{L}}{\partial t}=\lambda_{0} \mu_{0} \frac{\partial^{2} \mathbf{A}_{l}}{\partial t^{2}}-\nabla \cdot \mathbf{A}_{l}=\mu_{0} \mathbf{J}_{l} \\
& \nabla \times \mathbf{B}_{T}-\epsilon_{0} \mu_{0} \frac{\partial \mathbf{E}_{T}}{\partial t}=\epsilon_{0} \mu_{0} \frac{\partial^{2} \mathbf{A}_{t}}{\partial t^{2}}+\nabla \times \nabla \times \mathbf{A}_{t}=\mu_{0} \mathbf{J}_{t}
\end{aligned}
$$


The constant, $\phi_{0}$, is called the electric polarizability of vacuum, and its unit is $\mathrm{Fs}^{2} \mathrm{~m}^{-3}$. In case of a stationary current potential $\left(\partial_{t} \mathbf{A}=\mathbf{0}\right)$, the GCED equations (3.20-3.21) reduce to the GMS equations (2.12-2.13), such that GCED will obey N3LM for open circuit stationary current distributions, by deducing the correct force theorem later on. The following inhomogeneous field wave equations can be derived from (3.1-3.3) and (3.19-3.21).

$$
\begin{aligned}
\frac{\phi_{0}}{\epsilon_{0}} \frac{\partial^{2} \mathbf{E}_{\Phi}}{\partial t^{2}}-\nabla \cdot \mathbf{E}_{\Phi} & =-\frac{1}{\epsilon_{0}} \nabla \rho \\
\frac{\phi_{0}}{\epsilon_{0}} \frac{\partial^{2} B_{\Phi}}{\partial t^{2}}-\nabla \cdot \nabla B_{\Phi} & =-\frac{1}{\epsilon_{0}} \frac{\partial \rho}{\partial t} \\
\lambda_{0} \mu_{0} \frac{\partial^{2} \mathbf{E}_{L}}{\partial t^{2}}-\nabla \cdot \mathbf{E}_{L} & =-\mu_{0} \frac{\partial \mathbf{J}_{l}}{\partial t} \\
\lambda_{0} \mu_{0} \frac{\partial^{2} B_{L}}{\partial t^{2}}-\nabla \cdot \nabla B_{L} & =-\mu_{0} \nabla \cdot \mathbf{J}_{l} \\
\epsilon_{0} \mu_{0} \frac{\partial^{2} \mathbf{E}_{T}}{\partial t^{2}}+\nabla \times \nabla \times \mathbf{E}_{T} & =-\mu_{0} \frac{\partial \mathbf{J}_{t}}{\partial t} \\
\epsilon_{0} \mu_{0} \frac{\partial^{2} \mathbf{B}_{T}}{\partial t^{2}}+\nabla \times \nabla \times \mathbf{B}_{T} & =\mu_{0} \nabla \times \mathbf{J}_{t}
\end{aligned}
$$

These wave equations describe the Transverse Electromagnetic (TEM) wave and two types of longitudinal electric waves. One type of longitudinal electric wave is expressed only in terms of the electric charge potential $\Phi$, so it is not induced by electric currents, see (3.22-3.23). It will be called a $\Phi$-wave. The second type of longitudinal electric wave is associated with the curl free electric current potential, see (3.24-3.25), and it will be called a Longitudinal Electromagnetic (LEM) wave. The following notations for the phase velocities of these wave types is used.

$$
a=\sqrt{\frac{\epsilon_{0}}{\phi_{0}}}, \quad b=\sqrt{\frac{1}{\lambda_{0} \mu_{0}}}, \quad c=\sqrt{\frac{1}{\epsilon_{0} \mu_{0}}}
$$

Initially we assume that the values of these phase velocities are independent constants, and that is why we introduced the new constants $\lambda_{0}$ and $\phi_{0}$. The additional theoretical prediction of the LEM wave and the $\Phi$-wave are testable, as before Maxwell's TEM wave prediction was tested by Hertz.

\subsection{General power- and force theorems}

Three power- and force laws can be derived that are associated with the $\Phi$-wave, the LEM wave and the TEM wave. The power- and force law for the $\Phi$-wave fields $B_{\Phi}$ and $\mathbf{E}_{\Phi}$ are derived from (3.1) and (3.19):

$$
\begin{aligned}
-\rho B_{\Phi} & =\frac{\phi_{0}}{2} \frac{\partial B_{\Phi}^{2}}{\partial t}+\frac{\epsilon_{0}}{2} \frac{\partial E_{\Phi}^{2}}{\partial t}-\epsilon_{0} \nabla \cdot\left(B_{\Phi} \mathbf{E}_{\Phi}\right) \\
\rho \mathbf{E}_{\Phi} & =\phi_{0}\left(\nabla B_{\Phi}\right) B_{\Phi}+\epsilon_{0}\left(\nabla \cdot \mathbf{E}_{\Phi}\right) \mathbf{E}_{\Phi}-\phi_{0} \frac{\partial\left(B_{\Phi} \mathbf{E}_{\Phi}\right)}{\partial t}
\end{aligned}
$$

The power- and force law for the LEM wave fields $B_{L}$ and $\mathbf{E}_{L}$ are derived from (3.2) and (3.201):

$$
\begin{aligned}
-\mathbf{E}_{L} \cdot \mathbf{J}_{l}= & \frac{1}{2 \mu_{0}} \frac{\partial B_{L}^{2}}{\partial t}+\frac{\lambda_{0}}{2} \frac{\partial E_{L}^{2}}{\partial t}-\frac{1}{\mu_{0}} \nabla \cdot\left(B_{L} \mathbf{E}_{L}\right) \\
B_{L} \mathbf{J}_{l}= & \frac{1}{\mu_{0}}\left(\nabla B_{L}\right) B_{L}+\lambda_{0}\left(\nabla \cdot \mathbf{E}_{L}\right) \mathbf{E}_{L} \\
& -\lambda_{0} \frac{\partial\left(B_{L} \mathbf{E}_{L}\right)}{\partial t}
\end{aligned}
$$

The power- and force law for the TEM wave fields $\mathbf{B}_{T}$ and $\mathbf{E}_{T}$ are derived from (3.3) and (3.21):

$$
\begin{aligned}
-\mathbf{E}_{T} \cdot \mathbf{J}_{t}= & \frac{1}{2 \mu_{0}} \frac{\partial B_{T}^{2}}{\partial t}+\frac{\epsilon_{0}}{2} \frac{\partial E_{T}^{2}}{\partial t} \\
& -\frac{1}{\mu_{0}} \nabla \cdot\left(\mathbf{B}_{T} \times \mathbf{E}_{T}\right) \\
\mathbf{B}_{T} \times \mathbf{J}_{t}= & \frac{1}{\mu_{0}} \mathbf{B}_{T} \times \nabla \times \mathbf{B}_{T}+\epsilon_{0} \mathbf{E}_{T} \times \nabla \times \mathbf{E}_{T} \\
& -\epsilon_{0} \frac{\partial\left(\mathbf{B}_{T} \times \mathbf{E}_{T}\right)}{\partial t}
\end{aligned}
$$

Similar energy flux vectors as Poynting's vector for the TEM wave, $-\mathbf{B}_{T} \times \mathbf{E}_{T}$, can be defined for the $\Phi$-wave: $B_{\Phi} \mathbf{E}_{\Phi}$ (see the last term in (3.29) and in (3.30), and for the LEM wave: $B_{L} \mathbf{E}_{L}$ (see the last term in (3.31) and in (3.32). For very small values of $\phi_{0}$, the $\Phi$-wave contribution to momentum change becomes very small as well, and yet the $\Phi$-wave contribution to power flux might be substantial!

Notice that the fields in these power- and force theorems are not superimposed fields. The most general power- and force theorems should be expressed in terms of superimposed fields, and these are defined as follows:

$$
\begin{aligned}
\mathbf{E} & =\mathbf{E}_{\Phi}+\mathbf{E}_{L}+\mathbf{E}_{T} \\
\mathbf{E}^{*} & =\frac{1}{c^{2}} \mathbf{E}_{\Phi}+\frac{1}{b^{2}} \mathbf{E}_{L}+\frac{1}{c^{2}} \mathbf{E}_{T} \\
B & =\frac{1}{c^{2}} B_{\Phi}+B_{L} \\
B^{*} & =\frac{1}{a^{2}} B_{\Phi}+B_{L}
\end{aligned}
$$

The field equations (3.19-3.21) are rewritten in terms of these fields.

$$
\begin{aligned}
\nabla \cdot \mathbf{E}-\frac{\partial B^{*}}{\partial t} & =\frac{1}{\epsilon_{0}} \rho \\
\nabla B+\nabla \times \mathbf{B}_{T}-\frac{\partial \mathbf{E}^{*}}{\partial t} & =\mu_{0} \mathbf{J}
\end{aligned}
$$

General power- and force theorems follow from (3.39) and (3.40):

$$
\begin{aligned}
& -\mathbf{E} \cdot \mathbf{J}-c^{2} B \rho= \\
& \frac{1}{\mu_{0}}\left[\mathbf{E} \cdot \frac{\partial \mathbf{E}^{*}}{\partial t}+\mathbf{B}_{T} \cdot \frac{\partial \mathbf{B}_{T}}{\partial t}+B \frac{\partial B^{*}}{\partial t}\right] \\
& -\frac{1}{\mu_{0}} \nabla \cdot\left(\mathbf{B}_{T} \times \mathbf{E}+B \mathbf{E}\right) \\
& \rho \mathbf{E}+\mathbf{J} \times \mathbf{B}_{T}+\left(\frac{\epsilon_{0}}{\lambda_{0}} \mathbf{J}_{l}+\mathbf{J}_{t}\right) B^{*}= \\
& \epsilon_{0}(\nabla \cdot \mathbf{E}) \mathbf{E}+\frac{1}{\mu_{0}}(\nabla \times \mathbf{E}) \times \mathbf{E}^{*}+ \\
& \frac{1}{\mu_{0}}\left(\nabla B+\nabla \times \mathbf{B}_{T}\right) \times \mathbf{B}_{T}+ \\
& \left(\epsilon_{0} \nabla B_{\Phi}+\frac{\epsilon_{0}}{\lambda_{0} \mu_{0}} \nabla B_{L}+\frac{1}{\mu_{0}} \nabla \times \mathbf{B}_{T}\right) B^{*}- \\
& \epsilon_{0} \frac{\partial\left(\mathbf{E} B^{*}\right)}{\partial t}-\frac{1}{\mu_{0}} \frac{\partial\left(\mathbf{E}^{*} \times \mathbf{B}_{T}\right)}{\partial t}
\end{aligned}
$$


In (3.42) the expression $\left(\frac{\epsilon_{0}}{\lambda_{0}} \mathbf{J}_{l}+\mathbf{J}_{t}\right)$ has to equal $\mathbf{J}$ in order to deduce the force law of (2.10) that satisfies N3LM. Therefore, the following condition is generally true: $\lambda_{0}=$ $\epsilon_{0} \quad(b=c)$. Applying this condition, the general power- and force theorems become (if $b=c$ then $\mathbf{E}^{*}=\frac{1}{c^{2}} \mathbf{E}$ ):

$$
\begin{gathered}
-\mathbf{E} \cdot \mathbf{J}-c^{2} B \rho= \\
\frac{\epsilon_{0}}{2} \frac{\partial E^{2}}{\partial t}+\frac{1}{2 \mu_{0}} \frac{\partial B_{T}^{2}}{\partial t}+\frac{B}{\mu_{0}} \frac{\partial B^{*}}{\partial t} \\
-\frac{1}{\mu_{0}} \nabla \cdot\left(\mathbf{B}_{T} \times \mathbf{E}+B \mathbf{E}\right) \\
\rho \mathbf{E}+\mathbf{J} \times \mathbf{B}_{T}+\mathbf{J} B^{*}= \\
\epsilon_{0}((\nabla \cdot \mathbf{E}) \mathbf{E}+(\nabla \times \mathbf{E}) \times \mathbf{E})+ \\
\frac{1}{\mu_{0}}\left(\nabla B+\nabla \times \mathbf{B}_{T}\right) \times \mathbf{B}_{T}+ \\
\frac{1}{\mu_{0}}\left(\nabla B+\nabla \times \mathbf{B}_{T}\right) B^{*}- \\
\epsilon_{0} \frac{\partial\left(\mathbf{E} B^{*}+\mathbf{E} \times \mathbf{B}_{T}\right)}{\partial t}
\end{gathered}
$$

From the law of charge continuity and the two scalar field wave equations (see (3.23) and (3.25), and also apply $\lambda_{0}=$ $\left.\epsilon_{0}\right)$, the following scalar field condition is derived for GCED:

$$
\frac{1}{c^{2}} \frac{\partial^{2} B^{*}}{\partial t^{2}}-\nabla \cdot \nabla B=0
$$

In case of general magnetostatics, the scalar fields are independent of time, and (3.45) is reduced to $\nabla \cdot \nabla B=0$, which is fulfilled by Lorenz's condition $(B=0)$, see also (2.14). If $B=0$ then $B_{\Phi}=-c^{2} B_{L}$, and $B^{*}$ is expressed as $B^{*}=\left(1-c^{2} / a^{2}\right) B_{L}$. In case of general magnetostatics, we also have $\mathbf{E}=\mathbf{E}_{\Phi}$, so the power theorem and the force theorem for General Magnetostatics are the following two equations:

$$
\begin{gathered}
-\mathbf{E}_{\Phi} \cdot \mathbf{J}=\frac{\epsilon_{0}}{2} \frac{\partial E_{\Phi}^{2}}{\partial t}+\frac{1}{\mu_{0}} \nabla \cdot\left(\mathbf{E}_{\Phi} \times \mathbf{B}_{T}\right) \\
\rho \mathbf{E}_{\Phi}+\mathbf{J} \times \mathbf{B}_{T}+\left(1-\frac{c^{2}}{a^{2}}\right) \mathbf{J} B_{L}=\epsilon_{0}\left(\nabla \cdot \mathbf{E}_{\Phi}\right) \mathbf{E}_{\Phi} \\
+\frac{1}{\mu_{0}}\left(\left(\nabla \times \mathbf{B}_{T}\right) \times \mathbf{B}_{T}+\left(1-\frac{c^{2}}{a^{2}}\right)\left(\nabla \times \mathbf{B}_{T}\right) B_{L}\right) \\
-\epsilon_{0}\left(\left(1-\frac{c^{2}}{a^{2}}\right) \frac{\partial \mathbf{E}_{\Phi}}{\partial t} B_{L}+\frac{\partial \mathbf{E}_{\Phi}}{\partial t} \times \mathbf{B}_{T}\right)
\end{gathered}
$$

This shows that the energy flow carried by stationary currents (for example, from a battery to an energy dissipating resistor [18]) just depend on the 'static' electric field, $\mathbf{E}_{\Phi}$, and the vector magnetic field, $\mathbf{B}_{T}$, where stationary currents forces depend also on the scalar magnetic field, $B_{L}$.

\subsection{The Whittaker premise versus the Lorentz premise}

In case of general magnetostatic currents, the density of the scalar magnetic field force must equal $\mathbf{f}_{L}=\mathbf{J} B_{L}$, according to Whittaker's force law that satisfies N3LM. Hence, the factor $\left(1-c^{2} / a^{2}\right)$ in the GCED force theorem for general magnetostatics (3.47) must be approximately equal to 1 in order to fulfill N3LM. We conclude that $a \gg c$ must be generally true, and this is the crux of GCED theory. We will call the assumption, $a \gg c$, the Whittaker premise, after E.T. Whittaker. This premise should not be confused with the Coulomb "gauge" condition, $B_{L}=0$. GCED in the Whittaker premise is called GCED-WP.

The assumption $a=c\left(B^{*}=B\right)$ will be called the Lorentz premise, after H.A. Lorentz. This premise should not be confused with the Lorenz "gauge" condition: $B=$ 0 . GCED in the Lorentz premise (GCED-LP) is equivalent with the inconsistent MCED theory, and for this reason the Lorentz premise must be false in general. This is proven as follows: with $a=c\left(B^{*}=B\right)$, (3.45) becomes:

$$
\frac{1}{c^{2}} \frac{\partial^{2}(B)}{\partial t^{2}}-\nabla \cdot \nabla(B)=0
$$

This equation implies that the superimposed scalar field exists as a free field wave that isn't sourced by any charge current density distribution. If $B$ isn't sourced by anything, it simply does not exist, then we can set $B=0$. It is easy to verify that GCED-LP reduces to MCED, by setting $B=B^{*}=0$.

The scalar fields equation (3.45) is a physical condition for physical potentials and physical scalar fields, since the Whittaker premise is true. We have shown that MCED, and in particular the confusing indeterminacy of the potentials in the context of MCED can be avoided, by replacing the Lorentz premise for the Whittaker premise. In $\S 4.1$ we refer to the experiments that verify the Whittaker premise and falsify the Lorentz premise.

\subsection{Retarded potentials and retarded fields}

The potentials of GCED-WP are determinate and physical, such that a unique solution of charge-current potentials describes the physics of a particular charge- and current distribution. With $a \gg c$, and $b=c$, the scalar- and vector potentials are solutions of the following decoupled inhomogeneous wave equations:

$$
\begin{array}{rll}
\frac{1}{a^{2}} \frac{\partial^{2} \Phi}{\partial t^{2}}-\Delta \Phi & =\frac{1}{\epsilon_{0}} \rho \\
\frac{1}{c^{2}} \frac{\partial^{2} \mathbf{A}}{\partial t^{2}}-\Delta \mathbf{A} & =\mu_{0} \mathbf{J}
\end{array}
$$

The solutions of these wave equations are the following retarded potentials:

$$
\begin{aligned}
\Phi(\mathbf{x}, t) & =\frac{1}{4 \pi \epsilon_{0}} \int_{V^{\prime}} \frac{\rho\left(\mathbf{x}^{\prime}, t_{a}\right)}{r} \mathrm{~d}^{3} x^{\prime} \\
\mathbf{A}(\mathbf{x}, t) & =\frac{\mu_{0}}{4 \pi} \int_{V^{\prime}} \frac{\mathbf{J}\left(\mathbf{x}^{\prime}, t_{c}\right)}{r} \mathrm{~d}^{3} x^{\prime} \\
r=\left|\mathbf{x}-\mathbf{x}^{\prime}\right| \quad & t_{a}=t-\frac{r}{a} \quad t_{c}=t-\frac{r}{c}
\end{aligned}
$$

The four retarded fields, derived from these potentials, are: 


$$
\begin{aligned}
& B_{\Phi}(\mathbf{x}, t)= \frac{1}{4 \pi \epsilon_{0}} \int_{V^{\prime}} \frac{-\dot{\rho}\left(\mathbf{x}^{\prime}, t_{a}\right)}{r} \mathrm{~d}^{3} x^{\prime} \\
& B_{L}(\mathbf{x}, t)=\frac{\mu_{0}}{4 \pi} \int_{V^{\prime}}\left[\frac{\mathbf{J}_{l}\left(\mathbf{x}^{\prime}, t_{c}\right) \cdot \mathbf{r}}{r^{3}}+\frac{\dot{\mathbf{J}}_{l}\left(\mathbf{x}^{\prime}, t_{c}\right) \cdot \mathbf{r}}{c r^{2}}\right] \mathrm{d}^{3} x^{\prime} \\
& \mathbf{B}_{T}(\mathbf{x}, t)=\frac{\mu_{0}}{4 \pi} \int_{V^{\prime}}\left[\frac{\mathbf{J}_{t}\left(\mathbf{x}^{\prime}, t_{c}\right) \times \mathbf{r}}{r^{3}}+\frac{\dot{\mathbf{J}}_{t}\left(\mathbf{x}^{\prime}, t_{c}\right) \times \mathbf{r}}{c r^{2}}\right] \mathrm{d}^{3} x^{\prime} \\
& \mathbf{E}(\mathbf{x}, t)=\frac{1}{4 \pi \epsilon_{0}} \int_{V^{\prime}}\left[\frac{\rho\left(\mathbf{x}^{\prime}, t_{a}\right) \mathbf{r}}{r^{3}}+\frac{\dot{\rho}\left(\mathbf{x}^{\prime}, t_{a}\right) \mathbf{r}}{a r^{2}}-\frac{\dot{\mathbf{J}}_{l}\left(\mathbf{x}^{\prime}, t_{c}\right)}{c^{2} r}\right. \\
&\left.-\frac{\dot{\mathbf{J}}_{t}\left(\mathbf{x}^{\prime}, t_{c}\right)}{c^{2} r}\right] \mathrm{d}^{3} x^{\prime}
\end{aligned}
$$

We identify three near field terms, that fall off in magnitude by $r^{2}$, and six far field terms of the $\Phi$, LEM and TEM waves, that fall off in magnitude by $r$, so GCED-WP is far field consistent.

Beside Electrostatics and General Magnetostatics, we define two other types of restricted behavior. A charge-current distributions is Quasi Dynamic (QD) if it is assumed that $a \rightarrow \infty$. A charge-current distribution is Quasi Static (QS) if it is assumed that $a \rightarrow \infty \wedge c \rightarrow \infty$. For QD distributions, the retardation of the Coulomb field $\mathbf{E}_{\Phi}$, and the scalar potential $\Phi$, is unnoticed $\left(t_{a}=t\right)$. The length of the circuit is much smaller than the wavelength of the far $\Phi$-wave, such that detection of a far $\Phi$ potential gradient is impossible: the second term in (3.56) becomes zero. The induction law (3.1) is still needed, since the secondary field $B_{\Phi}$ does not disappear for quasi dynamics. QD is often referred to as 'instantaneous action at a distance' [19]. In case of QS, also the second term in (3.54) and (3.55) become zero. The induction laws for the secondary fields $\mathbf{E}_{L}$ and $\mathbf{E}_{T}$ are still required for quasi statics, since the third and fourth term in (3.56) do not disappear.

\subsection{The $\frac{4}{3}$ problem of MCED}

The electrostatic energy, $E_{e}$, and the electromagnetic momentum, $\mathbf{p}_{e}$, of an electron with charge $q_{e}$ (that is distributed on the surface of a sphere with classical electron radius, $r_{e}$ ) which has a constant speed $\mathbf{v}$, are the following expressions, derived from Maxwell's CED:

$$
\begin{aligned}
& E_{e}=\frac{1}{2} \frac{1}{4 \pi \epsilon_{0}} \frac{q_{e}^{2}}{r_{e}}=m_{e} c^{2} \\
& \mathbf{p}_{e}=\frac{2}{3} \frac{\mu_{0}}{4 \pi} \frac{q_{e}^{2}}{r_{e}} \mathbf{v}=m_{e}^{\prime} \mathbf{v}
\end{aligned}
$$

Notice the following inconsistency: $m_{e}^{\prime}=\frac{4}{3} m_{e}$, which is known as the $\frac{4}{3}$ problem in the context of MCED.

David E. Rutherford offered a solution to the this problem. His expressions for the electrostatic energy and the electromagnetic momentum are as follows:

$$
\begin{aligned}
& E_{e}=\frac{1}{4 \pi \epsilon_{0}} \frac{q_{e}^{2}}{r_{e}}=m_{e} c^{2} \\
& \mathbf{p}_{e}=\frac{\mu_{0}}{4 \pi} \frac{q_{e}^{2}}{r_{e}} \mathbf{v}=m_{e}^{\prime} \mathbf{v}
\end{aligned}
$$

Firstly, Rutherford [20] proves that the electrostatic energy of the electron is twice that of (3.57), since the work rate, in order to charge the electron sphere with radius $r_{e}$ with a charge $q_{e}$, is equal to $\partial_{t}\left(q_{e} \Phi_{e}\right)=\partial_{t}\left(q_{e}\right) \Phi_{e}+q_{e} \partial_{t}\left(\Phi_{e}\right)$, and not just equal to $\partial_{t}\left(q_{e}\right) \Phi_{e}$. If we consider only the net charge potential, $\Phi$, the GCED power density is equal to $\nabla \Phi \cdot \mathbf{J}+\partial_{t}(\Phi) \rho$. The volume integral of $\nabla \Phi \cdot \mathbf{J}$ equals $\Phi_{e} \partial_{t}\left(q_{e}\right)$. The volume integral of $\partial_{t}(\Phi) \rho$ equals $\partial_{t}\left(\Phi_{e}\right) q_{e}$, and therefore the energy flow of charging the electron sphere equals $\partial_{t}\left(q_{e} \Phi_{e}\right)$, such that (3.59) also follows from GCED.

This shows that the $\frac{4}{3}$ problem is in fact a $\frac{2}{3}$ problem: an electromagnetic momentum of $\frac{1}{3} m_{e} \mathbf{v}$ is missing. Secondly, Rutherford [21] derives the electromagnetic momentum (3.60) by means of the electromagnetic momentum density expression $\epsilon_{0}\left(\mathbf{E} B_{L}+\mathbf{E} \times \mathbf{B}_{T}\right)$; the volume integral of the electromagnetic momentum density $\epsilon_{0}\left(\mathbf{E} B_{L}\right)$ is equal to the missing $\frac{1}{3} m_{e} \mathbf{v}$ momentum. The time derivative of this momentum density expression is exactly the last term of (3.44) in case we apply the Whittaker premise $\left(B^{*}=B_{L}\right)$, thus (3.60) follows also from GCED-WP.

Now we have $m_{e}=m_{e}^{\prime}$ without factor $\frac{4}{3}$, and this means that the famous equation $E=m c^{2}$ can be derived from the non-relativistic GCED-WP theory by evaluating the static energy and the electromagnetic momentum of a charged sphere in uniform motion. The consistent GCED-WP theory does not suffer the $\frac{4}{3}$ problem either.

\section{Review of CED experiments}

The development of GCED-WP was motivated by Nikola Tesla's [22, 23] remarkable achievements in electrical engineering. Tesla described his long distance electric energy transport system as transmission of longitudinal electric waves, conducted by a single wire or the natural media, including the aether. Mainstream physics predicts that longitudinal electric waves exist as sound waves conducted by material media only. GCED-WP predicts luminal longitudinal electromagnetic waves, and super luminal electric $\Phi$-waves in vacuum as well, hence, for the first time in history Tesla's observation of a longitudinal aether sound wave is supported by an exact theory. The characteristic 'quarter wave length' distribution, across the unwound wire length of the secondary coil of Tesla's transformer device, is hard to explain by conventional electrodynamics theory, where GCEDWP explains this wave type naturally as a LEM wave. A most general review is required for a wide range of CED experiments that may verify or falsify the new aspects of GCEDWP theory.

\subsection{The superluminal Coulomb field}

SCED and GCED-WP predict that the Coulomb field is superluminal. Superluminal evanescent 'tunneling' of fields has been reported [24]. Usually such effects are explained as quantum effects, however, GCED-WP $(a \gg c)$ explains such effects as a Coulomb near field with superluminal speed, see also [25]. The authors of [26] experimentally proved that the Coulomb near field of a uniformly moving electron beam is rigidly carried by the beam itself, which is further described as follows: the Coulomb near field travels with velocity much greater than $c$. It is impossible to explain these results by means of MCED, since Jefimenko's electric field expression predicts a field retardation time interval of $r / c$ for the electric near field and the electric far field. 


\subsection{General Magnetostatic force experiments}

The historic Magnetostatic force experiments carried out by Ampère, Gauss, Weber and other famous scientist, are examples of open circuit currents. It is certain that batteries or very large capacitor banks were used as electric current sources and current sinks that typically showed time varying charge densities and divergent currents at the current source/sink interface, however, delivered a steady voltage and a stationary current.

Specific General Magnetostatic experiments such as Ampère's historic hairpin experiment, demonstrate the existence of the longitudinal Ampère force [27] [28] [9]. In particular, Stefan Marinov [8] and Genady Nikolaev [13] published on the results of several GMS force experiments that prove the existence of longitudinal Ampère forces. Nikolaev suggested a classical explanation for the Aharonov Bohm effect: it is a longitudinal Ampère force, acting on the free electrons that pass through a double slit and pass a shielded solenoid on both sides of the solenoid. Such a force does not deflect the free electrons, and it slightly decelerate (delay) or accelerate (advance) the electrons, depending on which side the electrons pass the solenoid, which explains classically the observed phase shift in the interference pattern.

An excellent example of General Magnetostatics is Marinov's stationary current motor that works as claimed, as observed by Phipps [29] and others. The Marinov motor configuration is very similar to the Aharonov Bohm experiment: two stationary electric currents, conducted by a metallic rotor ring, pass a solenoid at both sides, such that only a longitudinal Ampère force can explain the ring rotation. Marinov referred to Whittaker's force law and Newton's third law of motion, in order to explain the ring rotation. Wesley's theory of the Marinov motor is incorrect, because equation $i=\mathbf{v} q$ has been applied incorrectly for the movement of net electric charge, $q$, in the conducting ring and the net electric current, $i$, in the conducting ring.

\subsection{Nikolaev induction}

Experiments have yet to be done to verify or falsify Nikolaev's induction law, see eq. 3.2. According to this law, primary sinusoidal divergent currents induce a secondary sinusoidal divergent electric field $\mathbf{E}_{L}$ and similar secondary currents (depending on the resistance in the secondary 'circuits'), such that the secondary current is 90 degrees (or more) out of phase with the primary currents.

\subsection{LEM waves}

Wesley and Monstein published a paper on the transmission of a $\Phi$-wave, by means of a pulsating surface charge on a centrally fed ball antenna [30]. They assumed divergent currents are not present in such an antenna $(\nabla \cdot \mathbf{J}=0)$, however, this suggests a violation of charge conservation $(\nabla \cdot \mathbf{J}=0$ and $\left.\partial_{t} \rho \neq 0\right)$. We suggest that a centrally fed ball antenna conducts curl free divergent currents that induce mainly LEM waves (the field $B_{\Phi}$ ] is negligible), and that Wesley and Monstein actually observed LEM waves in stead of $\Phi$-waves. They tested and confirmed the longitudinal polarity of the received electric field.

Ignatiev and Leus used a similar ball shaped antenna to send wireless longitudinal electric waves with a wavelength of $2.5 \mathrm{~km}$ [31]. They measured a phase difference between the wireless signal and an optical glass fiber control signal (the two signals are synchronous at the sender location) at a $0.5 \mathrm{~km}$ distance from the sender location. They concluded from the measured phase shift that the wireless signal is faster than the optical glass fiber signal, and that the wireless signal has a phase velocity of $1.12 \mathrm{c}$. However, we assume that the $0.12 c$ discrepancy is due to an incorrect interpretation of the data, for instance, the optical glass fiber control signal has a phase velocity slower than c (in most cases it is 200,000 $\mathrm{km} / \mathrm{sec}$, depending on the refractive index of the glass fiber). Combining the results from the experiments by Wesley, Monstein, Ignatiev and Leus, we conclude that the results verify the existence of the LEM wave that has luminal speed $c$ in air.

A very efficient 'quasi-superconducting' Single Wire Electric Power System (SWEP) has been tested [32], that meets the same power requirements for standard $50 / 60 \mathrm{~Hz}$ threephase AC power lines. The SWEP system transmits a high frequency high voltage signal that is send and received by tuned and synchronized Tesla transformers. 'Floating' SWEP applications are described that do not require grounding of the SWEP system, therefore it is reasonable to assume the SWEP system is based on the LEM wave concept. Single wire transmission systems that transport TEM wave energy are 'ground return' systems that always require ground connections, such that the electric field is perpendicular to the wire and ground.

\subsection{The $B_{\Phi}$ field and $\Phi$-waves}

The field $B_{\Phi}$ only exists as far field, so observable effects of this type of field are not similar to near field force interaction, but through the emission and reception of $\Phi$-wave energy. In order to induce observable $B_{\Phi}$ fields, one needs to induce high electric charge potentials with very high frequencies. High $B_{\Phi}$ fields may be induced by means of collective tunneling of many electrons through a potential energy barrier, since the tunneling of electrons is practically instantaneous. The $\Phi$-wave energy flux vector $\epsilon_{0} B_{\phi} \mathbf{E}_{\Phi}$ also depends on the magnitude of electric fields, which can be optimized as well. Negative Resistance Oscillators (NROs) and Negative Conductance Oscillators (NCOs) may be suitable senders and receivers of continuous $\Phi$ waves; such oscillators induce the highest electric potential frequencies, and are either based on avalanche ionization effects or collective quantum tunneling effects (or both).

Podketnov's "impulse gravity" generator emits a far field signal pulse with velocity of at least $64 c$ [33], over a distance of 1211 meter. The wireless pulse is generated by means of a high voltage discharge (maximum of 2 million volt) from a superconducting flat surface electrode to another nonsuperconducting electrode. The emitted pulse travels into the direction longitudinal (parallel) to the electronic discharge direction. TEM wave radiation, transverse to the direction of discharge, was not detected. Podkletnov concluded that the 'longitudinal direction' signal isn't a TEM wave, nor a beam of mass particles. We assume that Podkletnov's impulse 'gravity' device generates $\Phi$-waves; the measured signal speed of at least $64 c$ agrees with the GCED-WP prediction of a superluminal $\Phi$-wave phase velocity $(a \gg c)$. Secondly, Podkletnov expects that the superluminal signal frequency matches the tunneling frequency of the discharged electrons [34]; during the discharge pulse, many electrons 
tunnel collectively through many superconducting layers before leaving the superconductor. This implies that a high $B_{\Phi}$ scalar field is induced, since electron tunneling is an almost instantaneous electronic effect. The electrodynamic nature of the impulse gravity force has not yet been fully investigated by Podkletnov and Modanese. This recently discovered force could also be a novel 'impulse dia-electric' force, that is repulsive for a wide range of materials, similar to the repulsive diamagnetic force in a strong magnetic field.

The reception of $\Phi$-waves is most likely the reverse process of the transmission of $\Phi$-waves, and requires the fastest movement of electrons, such as quantum tunneling through an energy barrier. The hypothesis that $\Phi$-waves may stimulate quantum tunneling of electrons will be called the $\Phi$-wave electric effect, similar to the photo-electric effect of electron emissions by a metal surface that is exposed to TEM waves.

\subsection{Evidence for natural longitudinal electric waves as energy source}

The most important GCED-WP application may be the conversion of natural existing longitudinal electric far fields into useful electricity. Nikola Tesla was convinced that such an energy conversion is possible [22, 23]. In particular, the conversion of natural superluminal $\Phi$-waves can be of great importance, since a high frequency energetic $\Phi$-wave penetrates much deeper into the earth and earth atmosphere than electromagnetic waves, because of its relative long wavelength. We assume that a few "free energy" device that were invented in the electronic age, might actually function as claimed and make use of the $\Phi$-electric effect.

Dr. T. Henry Moray's radiant energy device converted the energy flow of natural 'cosmic aether' waves into $50 \mathrm{KWatt}$ of useful electricity, day and night [35]. Moray's device did not include batteries or large capacitor banks to store energy. Moray tuned his radiant energy device into a natural high frequency signal, that we assume is a $\Phi$-wave of natural origin. Dr. Harvey Fletcher, who was the co-discoverer of the elementary charge of the electron as the assistant of Nobel price winner Dr. Millikan, signed an affidavit [36] describing that Moray's radiant energy receiver functioned as claimed. The most proprietary component of Moray's device was a high voltage cold cathode tube that contained a Germanium electrode doped with impurities, called 'the detector tube' by Moray. The high voltage high frequency electric potential at the first energy receiving stage appeared to be at over 200,000 volts. Electron tunneling and avalanche ionization effects explain the observed high frequency signal generated by Moray's valve tube, as well as the reported negative slope in a part of the conduction characteristic of the Moray tube. Moray's suggestion that transverse electromagnetic gamma rays are the energy source of his device is unlikely, since the cosmic gamma ray intensity at the earth surface is too weak to explain $50 \mathrm{KWatt}$ of continuous output power generated by such a small device. The conversion of mass energy into electricity as alternative explanation for Moray's device power output was rejected by Moray himself, therefore $\Phi$-wave energy conversion remains one of the few explanations.

Dr. P.N. Correa's [37] energy conversion system is also based on a cold cathode plasma tube, which shows an excess electric power output. Correa described an anomalous and longitudinal cathode reaction force during self-pulsed abnormal glow discharges in a cold cathode plasma tube. Cor- rea observed the abnormal glow discharge in a negative slope current-voltage regime. The same 'pre-discharge' glow has been observed by Podkletnov, just before the pulse discharge of his 'gravity' impulse device. A similar excess energy result was achieved by Dr. Chernetsky [38] by means of a selfpulsed high voltage discharge tube filled with hydrogen gas. Chernetsky's hydrogen gas tube generated longitudinal electronic waves in the electrical circuits attached to the tube, powering several hundred watt lamps.

These self-oscillating electronic plasma tube systems are without doubt negative resistance/conductance oscillators (NRO/NCO), optimized for Fowler-Nordheim quantum tunneling from a cold cathode to vacuum, and optimized for avalanche effects. The self-oscillation criteria for NROs and NCOs are not fully understood even today. We suggest that this type of device can function as a powerful $\Phi$-wave energy receiver, which explains its excess energy output.

\section{Conclusions}

N3LM describes the motion of bodies that have mass; this law does not take into account the momentum of massless electromagnetic radiation. N3LM can be replaced by the more general principle of conservation of the sum of massand massless momentum. It is sometimes concluded that MCED satisfies the more general principle of momentum conservation, however, MCED violates this principle as well: circuits of stationary currents do not send or receive electromagnetic radiation with massless momentum, and it was already shown that Grassmann force law violate N3LM, in case of General Magnetostatics. One might take into account infrared radiation caused by friction, emitted by magnetostatic current circuits. However, infrared radiation does compensate for the non-reciprocal Lorentz force, since the magnitude of momentum changes due to infrared radiation usually is much smaller than the occurring Ampère forces exerted on conductors, and secondly, this radiation is usually emitted in all directions such that the contribution of momentum change due to infrared radiation emission nullifies.

The fundamental theorem of vector algebra, that holds for time dependent vector functions [39] as well, is essential for a comprehensive treatise and derivation of a consistent classical electrodynamics. We showed that GCED-LP $(a=c)$ reduces to the inconsistent MCED, that shows indeterminate potentials. Therefore, we conclude that the Lorentz premise is false, and that the Whittaker premise $(a \gg c)$ is fundamentally true. GCED-WP is a consistent electrodynamics theory, naturally based on the law of charge continuity (and not on divergence free currents), that solves the $\frac{4}{3}$ problem as well. Experimental results exist that verify GCEDWP and falsify MCED. GCED-WP and the extra condition $\nabla \cdot \mathbf{J}=\partial_{t} \rho=0$ reduces to SCED, which is a consistent theory and the essence of MCED. Classical electrodynamics is closely tied to modern physics theories, such as relativity theory and quantum mechanics.

The false Lorentz premise $(a=c)$ is key to understand the second postulate of Special Relativity (SR) theory, a proposition in the following circular arguments: MCED does not predict longitudinal wave modes in vacuum, therefore vacuum can not be a physical medium (a physical medium allows for longitudinal waves), therefore a relative motion of an observer with respect to vacuum is not possible, such that 
the speed of light has an absolute constant value $c$ regardless of the relative motion of light source and observer (the second SR postulate), such that the Lorentz transform has preference over the Galilei transform, which further forbids velocities higher than constant $c$, such that $a=c$, and this reduces GCED to MCED, etc ... These circular arguments of SR theory began with the false Lorentz premise $(a=c)$, which is the most fundamental assumption of SR, and which reduces GCED to the inconsistent MCED. One-way TEM wave experiments prove the anisotropy of the TEM wave velocity in vacuum [40], and the recent "gravity impulse" speed measurements by E. Podkletnov [33] is strong evidence for a superluminal wave speed of $64 c$. These experiments falsify both the Lorentz-Poincare SR theory and Hilbert's general relativity theory. To formulate a relativistic and consistent GCED-WP theory, a relativity principle other than Lorentz invariance should be applied anyway. Heinrich Hertz and Thomas E. Phipps [41] showed how to cast MCED into a Galilei invariant form by simply replacing the partial time differential operator by the total time differential operator. In this way GCED-WP can be cast into a relativistic Galilei invariant GCED-WP.

The unfounded conjecture from Quantum Mechanics theory: "the linearly dependent 'scalar' photon and 'longitudinal' photon do not contribute to field observables", is obviously based on the false Lorentz premise, and is expressed classically as follows: $B=B^{*}=0$. The indeterminacy of the quantum wave function, $\psi$, is tied to the indeterminacy of the MCED potentials, $\Phi$ and A. A gauge transform of the relativistic quantum wave equation is a transformation of the MCED potentials and the quantum wave function in $\Phi^{\prime}$, $\mathbf{A}^{\prime}$ and $\psi^{\prime}$, such that the phase of the transformed function $\psi^{\prime}$ differs a constant with the phase of the function $\psi$, and such that the relativistic quantum wave equation is "gauge invariant" [42]. This means the function $\psi$ is 'unphysical' and indeterminate as well. The indeterminacy of $\psi$ is explained as "probabilistic behavior" of elementary particles (the Born rule): the particle velocity is the $\psi$ wave group velocity, and is not associated with $\psi$ wave phase velocity. Gauge invariance is also known as gauge symmetry, and is supposed to be the guiding principle of modern particle physics, however, this "gauge freedom" is merely the consequence of a false Lorentz premise $(a=c)$, that reduces determinate GCED potentials to indeterminate MCED potentials. Recent scanning tunneling microscope experiments [43] falsify Heisenberg's uncertainty relations by close two orders of magnitude, which is proof for the deterministic physical nature of the quantum wave function. The determinate potentials of GCED-WP are agreeable with a determinate quantum wave function, where the phase of $\psi$ has physical meaning. The De Broglie-Bohm pilot wave theory comes into mind, in order to reinterpret quantum entanglement behavior as interferences of physical pilot waves. The unknown nature of Bohm's pilot wave has been an objection against pilot wave theory, ever since Bohm and de Broglie offered his interpretation Schrödinger's equation solutions. Caroline H. Thompson [44] published a paper on the universal $\psi$ function as the $\Phi$-wave aether. Indeed, super luminal $\Phi$-wave fields, acting as particle pilot waves, is a natural suggestion, such that the pilot wave nature is no longer "ghost like" and unknown. Quantum nonlocality and non-causal entanglement may be confused with causal and local quasi dynamics. GCED-WP offers a classical foundation for the elementary particle-wave duality. The electromagnetic momentum and the near $\Phi$-potential energy describes the particle mass energy and mass momentum, and the pilot $\Phi$-wave particle interference describes the particle wave nature.

Although a consistent physics foundation based on determinate functions is the final destiny of modern physics, it is of greater importance that GCED-WP inspires scientists and engineers to review past classical electrodynamics experiments, which may birth a new era of science and technology with respect to telecommunication and energy conversion.

\section{Acknowledgements}

We are grateful to Samer Al Duleimi and Ernst van Den Bergh for valuable discussions.

\section{REFERENCES}

[1] James Clerk Maxwell. A treatise on electricity and magnetism, volume One. Dover Publications, Inc. New York, 1954.

https://ia902302.us.archive.org/25/items/

ATreatiseOnElectricityMagnetism-Volume1/

Maxwell-ATreatiseOnElectricityMagnetismVolume1. pdf.

[2] James Clerk Maxwell. A treatise on electricity and magnetism, volume Two. Dover Publications, Inc. New York, 1954.

https://ia902302.us.archive.org/25/items/

ATreatiseOnElectricityMagnetism-Volume2/

Maxwell-ATreatiseOnElectricityMagnetismVolume2. pdf.

[3] Olivier Darrigol. Electrodynamics from Ampère to Einstein, page 210. Oxford University Press, 2003.

[4] Richard P. Feynman. The feynman lectures online. http://www.feynmanlectures. caltech.edu/II_13. html.

[5] John David Jackson. Classical Electrodynamics Third Edition. Wiley, 1998.

http://www. amazon.com/

Classical-Electrodynamics-Third-Edition

$\backslash$-Jackson/dp/047130932X.

[6] David J. Griffiths. Introduction to Electrodynamics. Pearson Education Limited, 2013.

http://www . amazon.com/

Introduction-Electrodynamics-Edition-।

\David-Griffiths/dp/0321856562.

[7] Alexander Altland. Classical electrodynamics. http://www.thp.uni-koeln.de/alexal/pdf/ electrodynamics.pdf.

[8] Stefan Marinov. DIVINE ELECTROMAGNETISM. EAST WEST International Publishers, 1993.

http://zaryad.com/wp-content/uploads/2012/10/ Stefan-Marinov.pdf.

[9] Jorge Guala-Valverde \& Ricardo Achilles. A manifest failure of Grassmann's force.

http://redshift.vif.com/JournalFiles/

V15NO2PDF/V15N2VAL. pdf. 
[10] E.T. Whittaker. THEORIES OF AETHER AND ELECTRICITY. Trinity College Dublin:printed at the University Press, by Ponsonby and Gibbs, 1910.

[11] A.K. Tomilin. The Fundamentals of Generalized Electrodynamics. D. Serikbaev East-Kazakhstan State Technical University, 2008. http://arxiv.org/ftp/arxiv/papers/0807/0807. 2172.pdf.

[12] David E. Rutherford. Action-reaction paradox resolution, 2006.

http://www. softcom.net/users/der555/actreact. pdf.

[13] Gennady V. Nikolaev. Electrodynamics Physical Vacuum. Tomsk Polytechnical University, 2004.

http://electricaleather.com/

d/358095/d/nikolayevg.v .

elektrodinamikafizicheskogovakuuma.pdf.

[14] Ludvig Valentin Lorenz. On the identity of the vibrations of light with electrical currents. Philosophical Magazine, 34:287-301, 1867.

[15] Michael Faraday. Experiments resulting in the discovery of the principles of electromagnetic induction. In Faraday's Diary, Volume 1, pages 367-92.

http://faradaysdiary.com/ws3/faraday.pdf, 1831.

[16] H.R Hertz. Ueber sehr schnelle electrische Schwingungen. Annalen der Physik, vol. 267, no. 7, p. 421-448, 1887.

[17] J.D. Jackson. From Lorenz to Coulomb and other explicit gauge transformations. Department of Physics and Lawrence Berkeley National Laboratory, LBNL-50079, 2002. http://arxiv.org/ftp/physics/papers/0204/ 0204034.pdf.

[18] Igal Galili and Elisabetta Goihbarg. Energy transfer in electrical circuits: A qualitative account.

http://sites.huji.ac.il/science/stc/staff_h/ Igal/Research\%20Articles/Pointing-AJP.pdf.

[19] Andrew E. Chubykalo and Roman Smirnov-Rueda. Instantaneous Action at a Distance in Modern Physics: "Pro" and "Contra" (Contemporary Fundamental Physics). Nova Science Publishers, Inc., 1999.

[20] David E. Rutherford. Energy density correction, 2003. http://www. softcom.net/users/der555/enerdens . pdf.

[21] David E. Rutherford. 4/3 problem resolution, 2002. http://www. softcom.net/users/der555/elecmass. pdf.

[22] Andre Waser. Nikola Tesla's Radiations and the Cosmic Rays.

http://www . andre-waser.ch/Publications/ NikolaTeslasRadiationsAndCosmicRays .pdf, 2000.

[23] Andre Waser. Nikola Tesla's Wireless Systems. http://www . andre-waser.ch/Publications/ NikolaTeslasWirelessSystems .pdf, 2000.

[24] G. Nimtz and A.A. Stahlhofen. Macroscopic violation of special relativity.

http://arxiv.org/ftp/arxiv/papers/0708/0708. 0681.pdf.

[25] Shuangjin Shi Qi Qiu Zhi-Yong Wang, Jun Gou. Evanescent Fields inside a Cut-off Waveguide as Near Fields. Optics and Photonics Journal, 2013, 3, 192-196, 1993.
[26] P. Patteri M. Piccolo G. Pizzella R. de Sangro, G. Finocchiaro. Measuring propagation speed of coulomb fields. http://arxiv.org/pdf/1211.2913v2.pdf.

[27] Lars Johansson. Longitudinal electrodynamic forces and their possible technological applications. Department of Electromagnetic Theory, Lund Institute of Technology, 1996. http://www.df.lth.se/ snorkelf/ LongitudinalMSc.pdf.

[28] Rémi Saumont. Mechanical effects of an electrical current in conductive media. 1. Experimental investigation of the longitudinal Ampère force. Physics Letters A, Volume 165, Issue 4, Pages 307-313, 25 May 1992, 1992.

[29] Thomas E. Phipps Jr. Observations of the Marinov motor. http://redshift.vif.com/JournalFiles/Pre2001/ V05NO3PDF/v05n3phi.pdf.

[30] C. Monstein and J.P. Wesley. Observation of scalar longitudinal electrodynamic waves. Europhysics Letters, 2002.

[31] V.A. Leus G.F. Ignatiev. On a superluminal transmission at the phase velocities. Instantaneous action at a distance in modern physics: "pro" and "contra", 1999.

[32] Stanislav V. Avramenko, Aleksei I. Nekrasov, Dmitry, S. Strebkov. SWEP SYSTEM FOR RENEWABLE-BASED ELECTRIC GRID. The All-Russian Research Institute for Electrification of Agriculture, Moscow, Russia, 2002. http://ptp.irb.hr/upload/mape/kuca/07_Dmitry_ S_Strebkov_SINGLE-WIRE_ELECTRIC_POWER_SYSTEM_ FOR_RE.pdf.

[33] Evgeny Podkletnov and Giovanni Modanese. Study of light interaction with gravity impulses and measurements of the speed of gravity impulses. In Gravity-Superconductors Interactions: Theory and Experiment, chapter 14, pages 169-182. Bentham Science Publishers, 2015. https://www.researchgate.net/publication/ 281440634_Study_of_Light_Interaction_with_ Gravity_Impulses_and_Measurements_of_the_ Speed_of_Gravity_Impulses.

[34] Evgeny Podkletnov and Giovanni Modanese. Investigation of high voltage discharges in low pressure gases through large ceramic superconducting electrodes.

http://xxx.lanl.gov/pdf/physics/0209051.pdf, 2003.

[35] Dr. T. Henry Moray. For Beyond the Light Rays Lies the Secret of the Universe. http://free-energy.ws/pdf/radiant_energy_1926. pdf, 1926.

[36] Harvey Fletcher. Affidavit, signed by dr. harvey fletcher. Affidavit, signed by Harvey Fletcher and Public Notary, confirming T.H. Moray's Radiant Energy Device functioned as claimed. http://thmoray.org/images/affidavit.pdf, 1979.

[37] Paulo N. Correa and Alexandra N. Correa. Excess energy (XS $N R G^{T M}$ ) conversion system utilizing autogenous pulsed abnormal glow discharge (PAGD). Labofex Scientific Report Series, 1996.

[38] A.V. Chernetsky. Processes in plasma systems with electric charge division. G.Plekhanov Institute, Moscow, 1989.

[39] Andrew Chubykalo, Augusto Espinoza, Rolando Alvarado Flores. Helmholtz theorems, gauge transformations, general covariance and the empirical meaning of gauge conditions. Journal of Modern Physics, 7:1021-1044, 2016. 
[40] Reginald T. Cahill. One-Way Speed of Light Measurements Without Clock Synchronisation.

http://www.ptep-online.com/index_files/2012/ PP-30-08.PDF, 2012.

[41] Thomas E. Phipps Jr. On hertz's invariant form of maxwell's equations.

http://www . angelfire.com/sc3/elmag/files/ phipps/phipps01.pdf.

[42] J. D. Jackson. Historical roots of gauge invariance. Department of Physics and Lawrence Berkeley National Laboratory,
LBNL-47066, 2001.

http://arxiv.org/pdf/hep-ph/0012061v5.pdf.

[43] Werner A. Hofer. Heisenberg, uncertainty, and the scanning tunneling microscope.

http://xxx.lanl.gov/pdf/1105.3914v3.pdf.

[44] Caroline H. Thompson. The phi-wave aether. In Has the Last Word Been Said on Classical Electrodynamics? -New Horizons, chapter 22, pages 350-370. Rinton Press, Inc, 2004. 